\title{
A rare case of primary cutaneous follicle centre lymphoma presenting as a giant tumour of the scalp and combined with JAK2V617F positive essential thrombocythaemia
}

\author{
Yordanka Tirefort ${ }^{1 *}$, Xuan-Cuong Pham ${ }^{2}$, Yasmine Lucile Ibrahim³ ${ }^{3}$, Thomas Pierre Lecompte ${ }^{1}$, Thomas Matthes ${ }^{1}$, \\ Christa Prins ${ }^{2}$, Begonia Cortes ${ }^{2}$, Michael Bernimoulin' ${ }^{1}, Y_{v e s}$ Chalandon ${ }^{1}$ and Kaveh Samii ${ }^{1}$
}

\begin{abstract}
Primary cutaneous follicle centre lymphoma (PCFCL) is a rare cutaneous B cell lymphoma in middle-age adults with excellent prognosis. Here we present a case of a patient with a PCFCL in the form of a giant tumour of the scalp in combination with a myeloproliferative neoplasm, JAK2V617F positive essential thrombocythaemia. This case may be of interest because of the favourable outcome in spite of the large size of the PCFCL, the rare combination with essential thrombocythaemia and because it contributes to discussion on the role of JAK2 mutation in such patients.
\end{abstract}

Keywords: Primary cutaneous follicle centre lymphoma, PCFCL, Essential thrombocythaemia, JAK2V617F, Myeloproliferative neoplasm

\section{Background}

Lymphoproliferative neoplasms (LPN) are a heterogeneous group of tumours of the lymphoreticular system that might manifest themselves as extranodal lymphomas, localized in organs or tissues. Primary cutaneous B-cell lymphoma is a distinct type of extranodal lymphomas with a particular clinicopathologic presentation [1]. Primary cutaneous follicle centre lymphoma (PCFCL) is the most common variant of cutaneous B-cell lymphomas, representing about $18 \%$ of all primary cutaneous lymphomas [2]. PCFCL usually manifests itself as variably sized, solitary or grouped erythematous lesions, localized in the head, neck, trunk, and upper extremities or, as in our patient, as multifocal lesions. Treatment almost always leads to complete remission but cutaneous relapses may occur.

Essential thrombocythaemia (ET) is a clonal myeloproliferative neoplasm (MPN) and JAK2V617F mutations are found in approximately $50 \%$ of ET patients. Only a few cases of concomitantly occurring MPN and LPN have been reported [3-8]. Laurenti et al. published

\footnotetext{
* Correspondence: Yordanka.Tirefort@hcuge.ch

'Department of Haematology, University Hospital of Geneva, Rue

Gabrielle-Perret-Gentil 4, 1211 Geneva, Switzerland

Full list of author information is available at the end of the article
}

a multicenter retrospective study on the association of chronic lymphocytic leukemia (CLL) and concomitant MPN [9] but at present, no systematic analysis for MPN-LPN disease association is available and underlying pathogenic mechanisms remain unclear. Here we present a patient with ET and a giant PCFCL tumour of the scalp treated with chemo- and radiotherapy.

\section{Case presentation}

A 56-year-old man with an unremarkable medical history developed a multinodular mass on the scalp reaching $19 \times 16 \times 4 \mathrm{~cm}$ over 18 months (Figure 1). Similar lesions appeared on the face. Skin biopsies showed a dense and diffuse infiltrate throughout the dermis and the subcutis of CD20, CD79a and Bcl-6 positive, CD10 and IRF4 negative, large lymphoid cells with a focal nodular growth pattern (Figure 2), an image typical of cutaneous follicle centre lymphoma with a diffuse growth pattern. IgH/Bcl-2 rearrangements were not detected using FISH. Cross-sectional PET/CT and MRI imaging did not reveal any evidence of systemic spread of the lymphoma except for a suspicious spinal lymph node on the right side of the neck (Figure 3). According to ISCL/EORTC classification of cutaneous lymphomas [1], a diagnosis of PCFCL, 

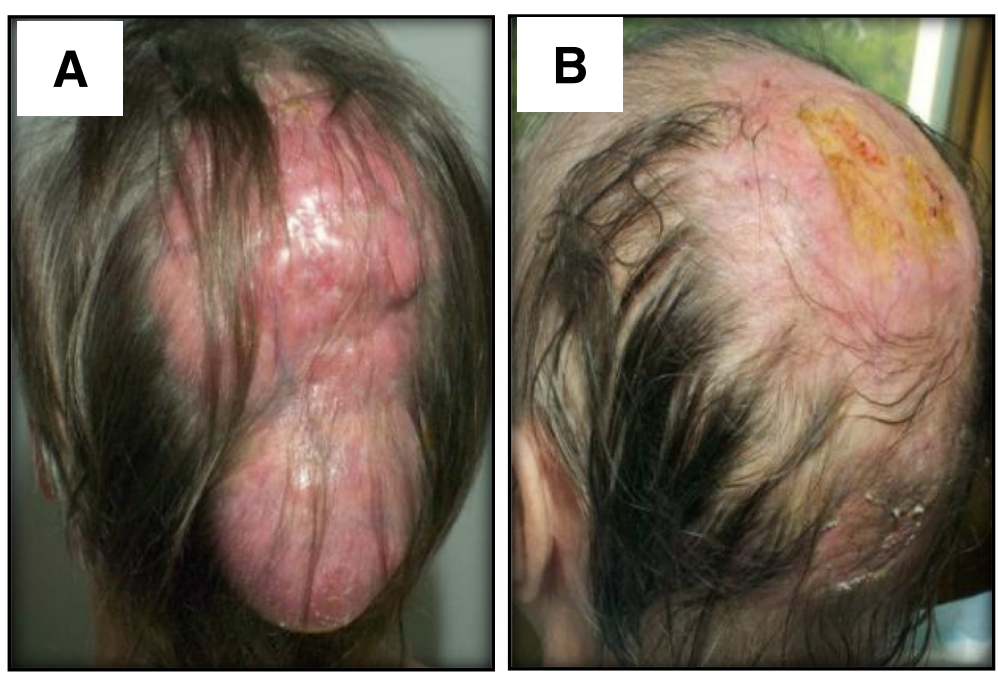

Figure 1 Multinodular mass on the scalp (A) and spectacular response after the second chemotherapy course (B).

stage T2bN1M0 was made. Platelet count was $772 \times 10^{9} / \mathrm{L}$, other blood counts were normal. LDH was normal and there was no splenomegaly. Bone marrow biopsy revealed a moderate hypercellularity with a marked proliferation of mature megakaryocytes with hyperlobulated nuclei, sometimes in loose clusters, without increase in reticulin fibres (Figure 2). Quantitative real-time PCR showed that $24 \%$ of the nucleated cells in the blood carried the JAK2V617F mutation, while scalp biopsies were only weakly positive $(<3 \%)$, probably due to granulocyte contamination. Screening for $\mathrm{HIV}, \mathrm{EBV}, \mathrm{HBV}$ and $\mathrm{HCV}$ was negative.

\section{Treatment}

Because of the exceptional size of the tumour, its location and possible nodal involvement, the patient received systemic chemotherapy consisting of 4 cycles of Rituximab combined with Cyclophosphamide, Adriamycin, Vincristine and Prednisone every 21 days (R-CHOP-21) followed by consolidation radiotherapy. After the second $\mathrm{R}$-CHOP cycle, we observed a spectacular response with $90 \%$ reduction of tumour mass (Figures 1B, 3E, 3F). At the time of writing, 2 years after diagnosis, the patient is still in remission. The chemotherapy did not induce a remission of ET and platelet counts remained stable at $600-700 \times 10^{9} / \mathrm{L}$ at 17 months after the last cycle of chemotherapy. Because the patient was without clinical symptoms of ET, no treatment other than a low dose prophylactic aspirin was given.

\section{Discussion}

We present herein a patient with PCFCL and a concomitant ET, two neoplasms of which the relation is still under debate [3-8]. For instance, Palandri et al. reported that 8 of 499 ET patients (1.6\%) also suffered from LPN
[6], while Rumi at al. found a 2.79-fold higher risk of developing LPN in patients with MPN than in the general population [8]. Similarly, Vannucchi et al. found a significantly increased risk of developing LPN in patients with MPN harbouring the JAK2V617F mutation, that varied from 2.7 fold for any LPN and ET, 4.2 fold for any LPN and PV to 12 fold in case of CLL and both ET and PV [7]. Because the JAK2V617F mutation was found in 2 of 3 LPN examined, they suggested that MPN and LPN might originate from a common lymphoid-myeloid progenitor. However, other studies did not report JAK2 mutations in the majority of LPN which underlines that JAK2V617F might only be one of the perhaps several factors favouring the genetic instability that predisposes to lymphoid and to myeloproliferative neoplasms as well $[3,10,11]$.

We have treated our patient with R-CHOP-21, which led to complete remission of the PCFCL without impacting the ET. This is reminiscent of the findings of Laurenti et al. who found that the course of MPN was not influenced by treating the coexisting CLL and that treating the MPN with hydroxyurea or imatinib did not impact the course of the LPN [9]. Although Palandri et al. did not find an increase in thrombotic events in their cohort of patients with concomitant ET and LPN [6], conventional chemotherapy for lymphoma might increase this risk considerably [12]. In the future, alternative treatment modalities, as those targeting the JAK/STAT pathway, might be useful because of their potential interest for treatment of both disorders in MPN-LPN patients. Downstream targets of the JAK2 pathway such as the signal transducer and activator of transcription (STAT) proteins, the Ras/Raf/ mitogen-activated protein kinases (MAPK), and the 

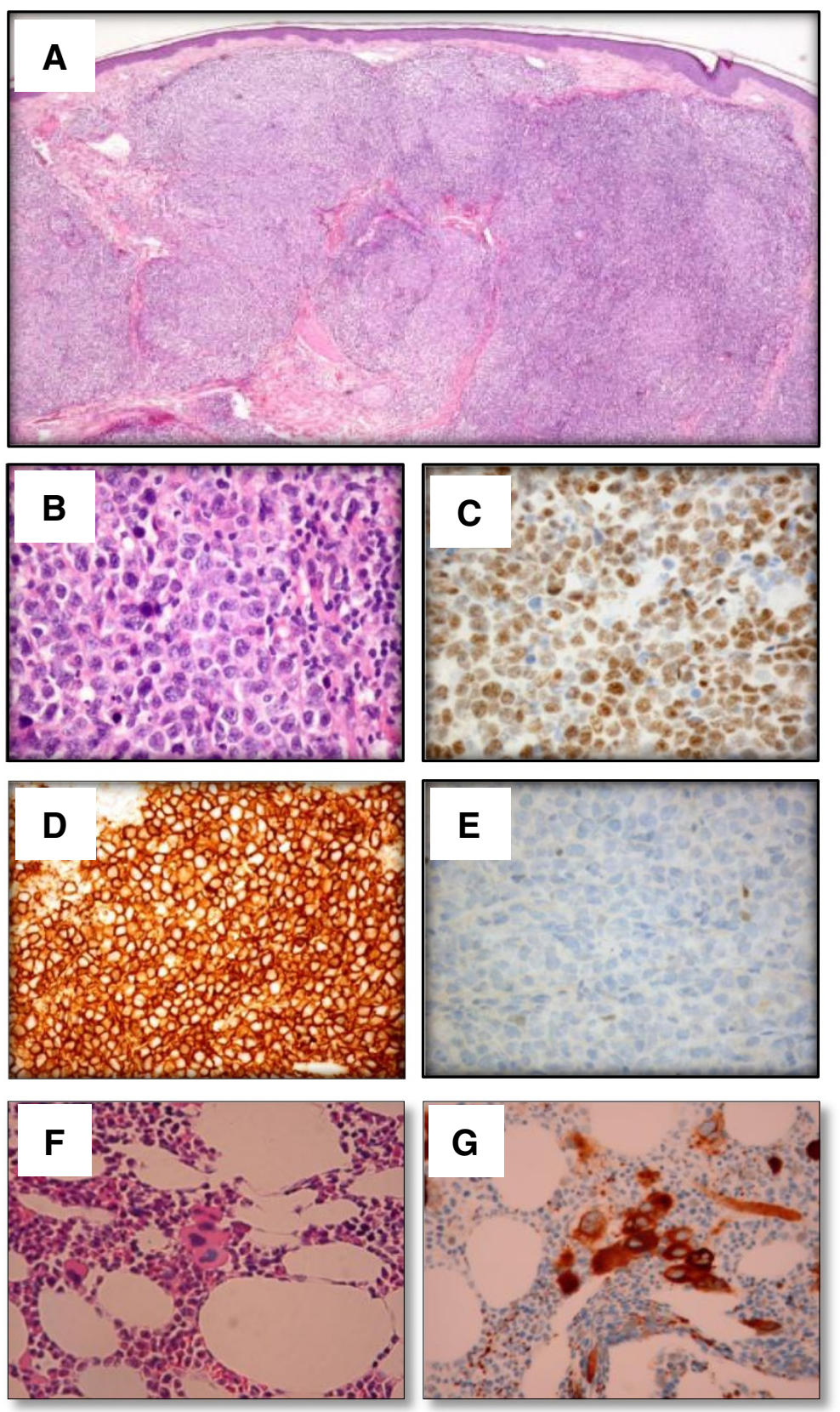

Figure 2 Histopatological fingings. Biopsy of the mass of the scalp showing a dense and diffuse dermal infiltrate with nodular growth pattern (A) consisted of large atypical lymphocytes (H\&E) (B), which are positive for CD20 (C) and Bcl6 (D) and negative for Bcl2 (E) on immunohistochemical staining. Bone marrow biopsy showing a hypercellular bone marrow with proliferation of megakaryocytes with hyperlobulated nuclei, sometimes in loose clusters (H\&E) (F), positive for CD61 on immunohistochemical staining (G).

phosphatidylinositol-3 kinase (PI3-K)/Akt pathways are often overexpressed in lymphoma subtypes such as primary mediastinal B-cell- and Hodgkin lymphoma [13-15]. Whether this is also true for the patient's PCFCL is not known. Unfortunately, we have not been able to test whether the JAK2V617F mutation in our patient had led to a sustained activation of the JAK2 pathway. If so, it would make sense to treat future MPN-LPN patients with the drugs targeting oncogenic JAK/STAT pathways that are currently under investigation for the treatment of myeloid and lymphoid neoplasms [16,17]. A recent phase I trial has already demonstrated a potential efficacy of the JAK2/FLT3 inhibitor Pacritinib (SB1518) for the treatment of different 

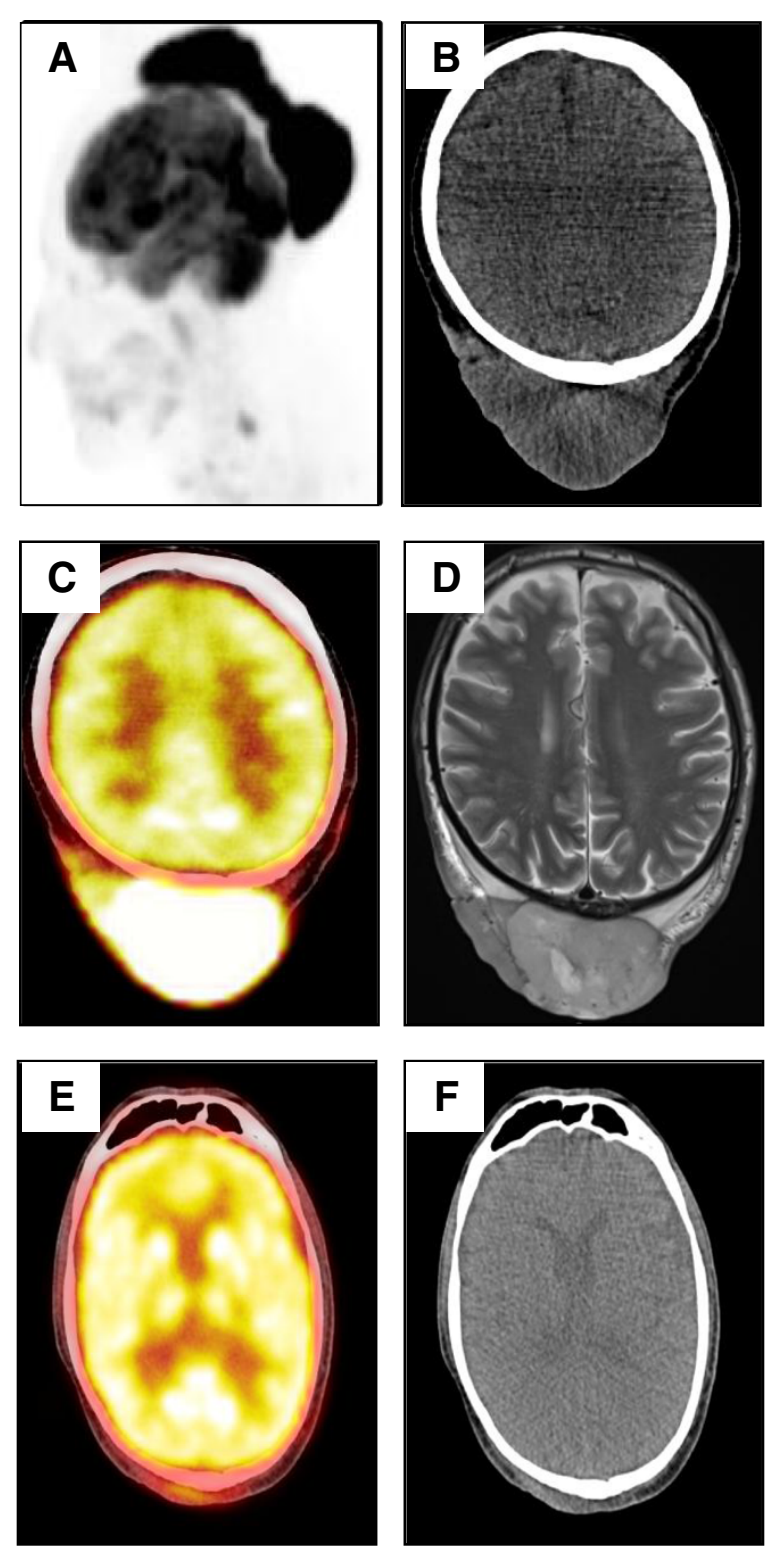

Figure 3 Radiological investigations showing the lesions before treatment and their evolution after the treatment. MRI at diagnosis (A) (B); PET-CT at diagnosis (C), (D); PET-CT and MRI after 2 cycles of R-CHOP (E), (F).

types of lymphoma reducing the tumour mass in half of the 31 patients enrolled with minimal haematological toxicity [18]. Furthermore, phase II studies of SB1518 in patients with myelofibrosis $[19,20]$ are now ongoing and if successful, this would certainly provide a rationale for its use in patients with concurring myeloid and lymphoid malignancies.

\section{Conclusion}

The present case is of interest because of the unusual presentation of PCFCL as a giant tumour of the scalp with multifocal lesions but nevertheless excellent evolution. To our knowledge, this is the first report of a patient with MPN, JAK2V617F positive ET, and PCFCL. The standard R-CHOP-21 therapy was successful for the PCFCL but this treatment did not influence the evolution of ET. In the future, therapies targeting JAK/STAT pathways that have shown potential for treatment of MPN as well as for lymphoma, may be considered an alternative.

\section{Consent}

Written informed consent was obtained from the patient for publication of this case report and the accompanying images. A copy of the written consent is available for review by the Editors-in-Chief of this journal. 


\section{Competing interest}

The authors declare no competing financial interest.

This case has been presented as a poster at the 81 th Annual assembly of Swiss Society of Internal Medicine in May 2013 in Basel, Switzerland.

\section{Authors' contributions}

$Y T, X C P, Y L I, T M$ and $C P$ diagnosed the patient. YT, MB, KS and BC treated and are following the patient. $Y T, T P L$ and $Y C$ wrote the manuscript. KS supervised the work. All authors reviewed the manuscript. All authors read and approved the final manuscript.

\section{Acknowledgments}

We thank Prof. Eddy ROOSNEK for his helpful suggestions and comments.

\section{Author details}

${ }^{1}$ Department of Haematology, University Hospital of Geneva, Rue Gabrielle-Perret-Gentil 4, 1211 Geneva, Switzerland. ${ }^{2}$ Department of Dermatology, University Hospital of Geneva, Rue Gabrielle-Perret-Gentil 4, 1211 Geneva, Switzerland. ${ }^{3}$ Department of Clinical Pathology, University Hospital of Geneva, Rue Gabrielle-Perret-Gentil 4, 1211 Geneva, Switzerland.

Received: 22 January 2014 Accepted: 29 March 2014

Published: 1 April 2014

\section{References}

1. Kim YH, Willemze R, Pimpinelli N, Whittaker S, Olsen EA, Ranki A, Dummer R, Hoppe RT: TNM classification system for primary cutaneous lymphomas other than mycosis fungoides and Sezary syndrome: a proposal of the International Society for Cutaneous Lymphomas (ISCL) and the Cutaneous Lymphoma Task Force of the European Organization of Research and Treatment of Cancer (EORTC). Blood 2007, 110:479-484.

2. Bouaziz JD, Bastuji-Garin S, Poszepczynska-Guigné E, Wechsler J, Bagot M: Relative frequency and survival of patients with primary cutaneous lymphomas: data from a single-centre study of 203 patients. $\mathrm{Br} J$ Dermatol 2006, 154:1206-1207.

3. Hauck G, Jonigk D, Kreipe $H$, Hussein K: Simultaneous and sequential concurrent myeloproliferative and lymphoproliferative neoplasms. Acta Haematol 2013, 129:187-196.

4. Gabrail NY, Martin TW: Coexistence of essential thrombocythemia and chronic lymphocytic leukemia. Acta Haematol 1991, 85:31-33.

5. Hernández-Boluda JC, Cervantes F, Alvarez A, López-Guillermo A, Montserrat E: Non-Hodgkin's lymphoma following untreated essential thrombocythemia. Leuk Lymphoma 2000, 36:421-423.

6. Palandri F, Derenzini E, Ottaviani E, Polverelli N, Catani L, Salmi F, Sabattini E, Bacci F, Zinzani PL, Baccarani M, Vianelli N: Association of essential thrombocythemia and non-Hodgkin lymphoma: a single-centre experience. Leuk Lymphoma 2009, 50:481-484.

7. Vannucchi AM, Masala G, Antonioli E, Chiara Susini M, Guglielmelli P, Pieri L, Maggi L, Caini S, Palli D, Bogani C, Ponziani V, Pancrazzi A, Annunziato F, Bosi A: Increased risk of lymphoid neoplasms in patients with Philadelphia chromosome-negative myeloproliferative neoplasms. Cancer Epidemiol Biomarkers Prev 2009, 18:2068-2073.

8. Rumi E, Passamonti F, Elena C, Pietra D, Arcaini L, Astori C, Zibellini S, Boveri E, Pascutto C, Lazzarino M: Increased risk of lymphoid neoplasm in patients with myeloproliferative neoplasm: a study of 1,915 patients. Haematologica 2011, 96:454-458.

9. Laurenti L, Tarnani M, Nichele I, Ciolli S, Cortelezzi A, Forconi F, Rossi D, Mauro FR, D'Arena G, Del Poeta G, Montanaro M, Morabito F, Musolino C, Callea V, Falchi L, Tedeschi A, Ambrosetti A, Gaidano G, Leone G, Foà R: The coexistence of chronic lymphocytic leukemia and myeloproliperative neoplasms: a retrospective multicentric GIMEMA experience. Am J Hematol 2011, 86:1007-1012

10. Lee JW, Soung YH, Kim SY, Nam SW, Park WS, Lee JY, Yoo NJ, Lee SH: JAK2 V617F mutation is uncommon in non-Hodgkin lymphomas. Leuk Lymphoma 2006, 47:313-314.

11. Wang YL, Lee JW, Kui JS, Chadburn A, Cross NC, Knowles DM, Coleman M: Evaluation of JAK2V617F in B and T cell neoplasms: identification of JAK2V617F mutation of undetermined significance (JMUS) in the bone marrow of three individuals. Acta Haematol 2007, 118:209-214.

12. Radaelli F, Onida F, Rossi FG, Zilioli VR, Colombi M, Usardi P, Calori R, Zanella A: Second malignancies in essential thrombocythemia (ET): a retrospective analysis of 331 patients with long-term follow-up from a single institution. Hematology 2008, 13:195-202.

13. Green MR, Monti S, Rodig SJ, Juszczynski P, Currie T, O'Donnell E, Chapuy B, Takeyama K, Neuberg D, Golub TR, Kutok JL, Shipp MA: Integrative analysis reveals selective 9p24.1 amplification, increased PD-1 ligand expression, and further induction via JAK2 in nodular sclerosing Hodgkin lymphoma and primary mediastinal large B-cell lymphoma. Blood 2010, 116:3268-3277.

14. O'Shea JJ, Holland SM, Staudt LM: JAKs and STATs in immunity, immunodeficiency, and cancer. N Engl J Med 2013, 368:161-170.

15. Derenzini E, Younes A: Targeting the JAK-STAT pathway in lymphoma: a focus on pacritinib. Expert Opin Investig Drugs 2013, 22:775-785.

16. Tam CS, Verstovsek S: Investigational Janus kinase inhibitors. Expert Opin Investig Drugs 2013, 22:687-699.

17. Furqan M, Mukhi N, Lee B, Liu D: Dysregulation of JAK-STAT pathway in hematological malignancies and JAK inhibitors for clinical application. Biomark Res 2013, 1:5-15.

18. Younes A, Romaguera J, Fanale M, McLaughlin P, Hagemeister F, Copeland A, Neelapu S, Kwak L, Shah J, de Castro Faria S, Hart S, Wood J, Jayaraman R, Ethirajulu K, Zhu J: Phase I study of a novel oral Janus Kinase 2 inhibitor, SB1518, in patients with relapsed lymphoma: evidence of clinical and biologic active ty in multiple lymphoma subtypes. J Clin Oncol 2012, 30:4161-4167.

19. Komrokji R, Wadleigh M, Seymour J, Roberts A, To L, Zhu H, Mesa R: Results of a phase 2 study of pacritinib (SB1518), a novel oral JAK2 inhibitor, in patients with primary, post-polycythemia vera, and post-essential thrombocythemia myelofibrosis [abstract]. ASH Annual Meeting Abstracts 2011, 118:282.

20. Deeg H, Odenike O, Scott B, Estrov Z, Cortes J, Thomas D, Zhu J, Kantarjian $H$, Verstovsek S: Phase II study of SB1518, an orally available novel JAK2 inhibitor, in patients with myelofibrosis [abstract]. J Clin Oncol 2011, 29(Suppl):6515.

doi:10.1186/2050-7771-2-7

Cite this article as: Tirefort et al:: A rare case of primary cutaneous follicle centre lymphoma presenting as a giant tumour of the scalp and combined with JAK2V617F positive essential thrombocythaemia. Biomarker Research 2014 2:7.

\section{Submit your next manuscript to BioMed Central and take full advantage of:}

- Convenient online submission

- Thorough peer review

- No space constraints or color figure charges

- Immediate publication on acceptance

- Inclusion in PubMed, CAS, Scopus and Google Scholar

- Research which is freely available for redistribution 